\title{
The role of phrase structure in the recall of meaningful verbal material'
}

JEREMY M. ANGLIN AND GEORGE A. MILLER HAR VARD UNIVERSTTY

Rote memorization of continuous prose passages was more rapid when the passages were presented in segments that conformed to the phrase structure of the sentences than when the segments violated that structure.

Since the work of Müller \& Schumann (1894) it has been clear that imposing a sequential pattern on a series of syllables affects the way it is memorized, and since Thorndike's (1931) experiments on 'belongingness" it has been clear that such effects can result from grammatical as well as from rhythmical groupings. Snygg (1935) demonstrated that segmentation of a passage into letter-triplets, ignoring the usual word boundaries, could greatly increase the difficulty of memorization. The present study is an extension of Snygg's work, but at the level of phrases, rather than individual words.

Method

Two coherent and meaningful paragraphs were constructed in such a way that every sentence was either simple, with a single noun phrase as subject and a verb phrase as predicate, or was compound with two such noun-phrase-verb-phrase pairs. These two paragraphs are given in full in the appendix, along with markers indicating the boundaries between successive phrases. The phrases contain from four to seven words. Each paragraph contains 32 phrases (16 noun and 16 verb phrases); one passage has 162 words, the other 169 .

The paragraphs were typewritten and presented on a Lafayette memory drum. Each paragraph was typed in two different ways, which yielded two different sets of materials. In the first set, the phrasesegmented material, each phrase was on a separate line, and thus appeared intact in the window of the memory drum. The second set, the nonphrase-segmented material, was prepared in exactly the same way, except that the sentences were always segmented exactly two words to the left of the phrase boundaries, so that fragments of two successive phrases were typed on a single line and appeared together in the window of the memory drum. Thus, except for the first and last lines, the number of words per line for a given paragraph was the same for the phrasesegmented version as for the nonphrase-segmented.

Twelve Harvard summer school students memorized the passages. They were randomly assigned to four treatments, with the restriction that there be three Ss per treatment. Each was tested twice, at intervals of from one to three days. The four treatments were:
(1) first learn paragraph $\mathrm{A}$ in phrase segments, later learn paragraph $B$ in nonphrase segments; (2) first $B$ in phrases, then $A$ in nonphrases; (3) first $A$ in nonphrases, then $B$ in phrases; and finally (4) first $B$ in nonphrases and then $A$ in phrases. This design balanced the order of paragraphs and the order of segmentations, and every $\mathrm{S}$ served as his own control.

In a given experimental session $S$ was seated in front of the memory drum and instructed to read the passage aloud as the drum advanced. The paragraph was presented at a rate of $2 \mathrm{sec}$ per phrase; the average reading rate was about 155 words per min, which is slightly faster than normal speech. When they had finished reading the entire paragraph, they wrote down, on paper provided for the occasion, "word for word" all they remembered of the paragraph. Six successive reading and writing trials comprised an experimental session, which lasted for about $50 \mathrm{~min}$.

\section{Results}

The measure of recall was the number of words in the original passage that appeared in the $S^{\prime} s$ protocol, regardless of order. This number was counted for each $\mathrm{S}$ on each trial.

Since our central interest is in the segmentation effect, we have averaged over Ss, paragraphs, and orders to obtain the functions shown in Fig. 1, where the percentage of words recalled is plotted for the two types of segmentation as a function of the trial number. Analysis of variance showed that the segmentation effect was significant $(F=11.98, \mathrm{df}=1 / 8$, $p<0.01)$. Neither the paragraph nor the order effect was significant.

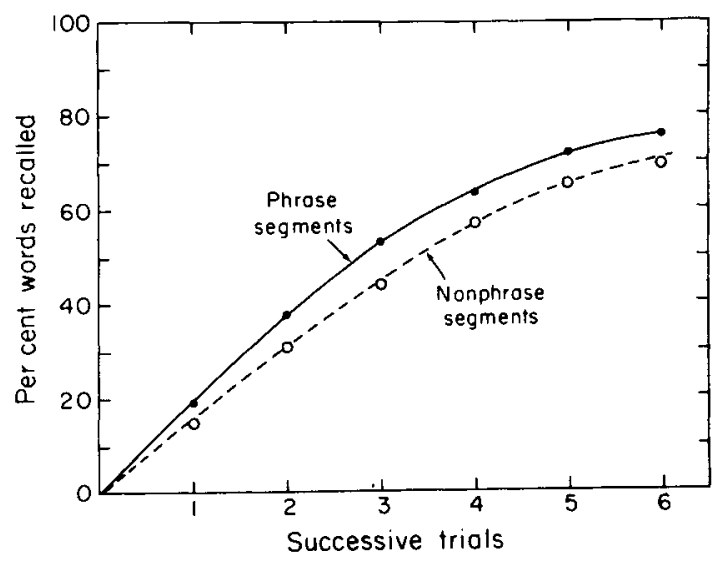

Fig. 1. 


\section{Discussion}

As expected, performance was slightly but significantly superior when the paragraphs were segmented in conformity with the phrase boundaries. The difference between the two functions in Fig. 1 is about $6 \%$, which means that the average $S$ recalled about 10 words more under phrase than nonphrase segmentation.

We interpret this result as supporting the psychological validity of phrase structure. We assume that, in order to read and remember the passages, Ss must grasp the grammatical structure of the sentences they contain. When the grammatical units are fragmented by a nonphrase type of segmentation, $S$ is forced to do additional work in order to recover them, and this additional work either distracts or subtracts from his memorization of the paragraph.

References

MÜLLER, G. E., \& SCHUMANN, F. Experimentelle Beiträge zur Untersuchung des Gedächtnisses. Z. Psychol, 1894, 6, 81-190, 257-339.

SNYGG, D. The relative difficulty of mechanically equivalent tasks: I. Human learning. J. genet. Psychol., 1935, 47, $299-319$.

THORNDIKE, E. L. Human learning. New York: Century, 1931. Note

1. This research was supported in part under Contract SD-187 between the Department of Defense, Advanced Research Projects Agency and Harvard University. We are indebted to David Cross for assistance with the statistical analysis.

\section{Appendix}

Passage A

The Union Army veterans / were garbed in blue and brass / and most of them / wore black slouch hats. / Their long kinky beards / were streaked with silver, / their jubilant, ringing laughter / echoed loudly and incessantly, / and the sound of many drums / boomed with implacable reassurance. / The Grand Army of the Republic / had arrived for its annual Encampment. / Each year this phenomenon / was witnessed with joviality and tears. / A kind of immaculate serenity / surrounded the Grand Army of the Republic / and something like pride / choked the heart with gladness. / For example, more than twenty thousand strangers / came to town on one occasion. / Crowded halls and lobbies / roared with tales of human triumph. / Pamphlets, cards, and posters / were distributed to all passers by. / A bombardment of drums / thundered beyond the gates of heaven, / and the sound of fifes / followed closely and surely behind. / The Pasadena Drum Corps / had tuned up outside the main entrance / and a volatile throng of people / rushed flocking to hear them. /

Passage B

Across the river the Michigan Drum Corps / was in the process of tuning up, / and the Michigan Drum Corps / was something to see. / The thickening exuberant crowds / shouted their favorite requests. / A clanging version of "Yankee Doodle" / appeared to appease them momentarily. / The crawling noisy traffic / pushed its way along the sidewalks. / Old grey bearded soldiers / limped unsteadily along everywhere. / A drum corps from Pittsburgh / played outside the Savory Hotel, / and a parading river of blue coats / marched up Locust Street. / Some infirm among the color-bearers / sought help from young boys, / but other determined and proud veterans / insisted on carrying weighty staffs alone. / An exuberant and thunderous applause / roared for these veterans like a waterfall. / The years of the annual Encampment / have long been gone. / The drumsticks and fifes of the veterans / have been stilled forever. / The men in black slouch hats / will march no more. / Yet the sound of their shuffling feet / lingers on and on / and we who have open ears / may still listen in rapture. 